\title{
Dynamics of a Stochastic SIRS Epidemic Model with Nonlinear and Saturated Incidence Rate
}

\author{
Jiying Ma* and Qing Yi \\ College of Science, University of Shanghai for Science and Technology, Shanghai, 200093, PR China \\ Email: majiying100@126.com
}

\begin{abstract}
The article is dedicated to the dynamics of a stochastic SIRS epidemic model, which is obtained by introducing Gaussian white noise to the transmission coefficient of a deterministic epidemic model with non-monotone and saturated incidence rate. The existence and uniqueness of positive global solution is proved for the stochastic model. The threshold parameter $R_{0}^{s}$ is established, and under some acceptable conditions the disease will go to extinction if $R_{0}^{s}<1$. However, the stochastic system has a unique ergodic stationary distribution and the disease is persistent if $R_{0}^{s}>1$. We also analyze the asymptotic behavior of the stochastic model near the disease-free equilibrium of the corresponding deterministic system. Numerical simulation is provided to support our theoretical results.
\end{abstract}

Keywords: stochastic epidemic model, extinction, asymptotic behavior, stationary distribution.

\section{Introduction}

Mathematical modeling has been considered as an important tool in the field of epidemiology and disease control. Since the classical $S I R$ and $S I S$ epidemic models established by Kermack and McKendrick in 1927, a great variety of epidemic models have been proposed and investigated $[7,8,9,18]$. For certain infectious diseases such as influenza, SIRS epidemic model is established in which recovered individuals continuously lose immunity to circulating viruses and return to susceptible class [10,16].

During the evolution of infectious diseases, the incidence rate of epidemic model plays a quite important role. In fact, the exact form of incidence rate is often difficult to obtain, because of the complex and fast-changing real environment. The traditional incidence rate is bilinear of the form $\beta S I$, which becomes unrealistic when the number of the infective individual gets large. Hence various forms of nonlinear incidence rate have been extensively used [1,6,12]. For instance, Liu et al. [11] studied the deterministic and stochastic $S I R S$ epidemic model with a nonlinear incidence $\frac{\beta S f(I)}{f(I)}$. Capasso and Serio [2] proposed a saturated incidence rate of the form $\frac{\beta S I}{1+\alpha I}$, where the infection function tends to a saturation level as the number of the infective individuals increases. Recently, to explain the phenomenon that the infection function first reaches the maximum value, then decreases and finally tends to saturation for certain infectious diseases, Lu et al. [13] adopted a generalized non-monotone and saturated incidence rate and analyzed the following $S I R S$ epidemic model

$$
\left\{\begin{array}{l}
\frac{d S(t)}{d t}=b-d S(t)-\frac{\beta S(t) I^{2}(t)}{1+k I(t)+\alpha I^{2}(t)}+\delta R(t), \\
\frac{d I(t)}{d t}=\frac{\beta S(t) I^{2}(t)}{1+k I(t)+\alpha I^{2}(t)}-(d+\mu) I(t), \\
\frac{d R(t)}{d t}=\mu I(t)-(d+\delta) R(t) .
\end{array}\right.
$$

where $S(t), I(t)$ and $R(t)$ denote the numbers of the population that are susceptible, infectious and removed, respectively. The parameter $b$ is the recruitment rate of the population; $d$ is the natural death rate of the population; $\mu$ denotes the natural recovery rate of infected members; $\delta$ is the rate at which recovered individuals lose immunity and return to the susceptible class; $\beta$ denotes the disease transmission rate; $\alpha$ is a parameter which measures the psychological or inhibitory effect; $k$ is a parameter such that $1+k I+\alpha I^{2}>0$ for all $I \geq 0$. In this paper, we further assume that all the parameters are positive. 
Furthermore, the transmission of infectious diseases is disturbed by various noises in the environment, such as the unpredictable contact with infected ones, population mobility and meteorological factors $[15,17]$. Therefore the parameters like birth rate, mortality rate, and transmission coefficient of the deterministic model may oscillate due to environmental fluctuations in the real world. Motivated by the approach in $[3,10]$, we assume that the transmission coefficient $\beta$ is subject to random environmental perturbation, that is, $\beta \rightarrow \beta+\sigma \xi(t)$, where $\xi(t)$ is the Gaussian white noise with mean zero and variance one. Hence, model (1) becomes

$$
\left\{\begin{array}{l}
d S(t)=\left[b-d S(t)-\frac{\beta S(t) I^{2}(t)}{1+k I(t)+\alpha I^{2}(t)}+\delta R(t)\right] d t-\frac{\sigma S(t) I^{2}(t)}{1+k I(t)+\alpha I^{2}(t)} d B(t), \\
d I(t)=\left[\frac{\beta S(t) I^{2}(t)}{1+k I(t)+\alpha I^{2}(t)}-(d+\mu) I(t)\right] d t+\frac{\sigma S(t) I^{2}(t)}{1+k I(t)+\alpha I^{2}(t)} d B(t), \\
d R(t)=[\mu I(t)-(d+\delta) R(t)] d t .
\end{array}\right.
$$

Note that $\xi(t) d t=d B(t)$, where $B(t)$ denotes the standard Brownian motion. We should mention that the infection function $\frac{\beta S(t) I^{2}(t)}{1+k I(t)+\alpha I^{2}(t)}$ does not satisfy the conditions of $(H 1)$ and $(H 3)$ in [10].

Throughout this paper, let $\left(\Omega, \mathcal{F},\left\{\mathcal{F}_{t}\right\}_{t \geq 0}, \mathbb{P}\right)$ be a complete probability space with a filtration $\left\{\mathcal{F}_{t}\right\}_{t>0}$ satisfying the usual conditions (i.e. it is increasing and right continuous while $\mathcal{F}_{0}$ contains all $\mathcal{P}$-null sets). $B(t)$ denotes a scalar Brownian motion defined on the complete probability space $\Omega$. Also let $\mathbb{R}_{+}^{3}=\left\{x_{i}>0, i=1,2,3\right\}$. In general, consider the following $d$-dimensional stochastic differential equation

$$
d X(t)=f(X) d t+\sum_{r=1}^{k} \sigma_{r}(X) d B_{r}(t)
$$

where $f(\cdot)$ is $\mathbb{R}^{d}$-valued function, $\sigma_{r}(\cdot)$ is $d \times m$-matrix-valued function, and $B_{r}(t), r=1, \ldots k$, are independent $m$-dimensional Brownian motions. Then the diffusion matrix is defined as follows

$$
A(x)=\left(a_{i j}(x)\right), \quad a_{i j}(x)=\sum_{r=1}^{k} \sigma_{r}^{i}(x) \sigma_{r}^{j}(x) .
$$

Furthermore, the differential operator $\mathcal{L}$ associated with equation (3) is defined by

$$
\mathcal{L} V(x)=\sum_{i=1}^{d} f_{i}(x) \frac{\partial V(x)}{\partial x_{i}}+\frac{1}{2} \sum_{i, j=1}^{d} a_{i j}(x) \frac{\partial^{2} V(x)}{\partial x_{i} \partial x_{j}}
$$

where $V(x)$ is an arbitrary twice continuously differential real-valued function.

The rest of the paper is organized as follows. In Section 2, the existence and uniqueness of positive solution is proved for system (2). In Section 3, we obtain the conditions for the extinction of the disease. In Section 4, we analyze the asymptotic behavior of the solution of system (2) near the disease-free equilibrium of the corresponding deterministic system. In Section 5, the sufficient conditions for the existence of a stationary distribution is established. In Section 6, numerical simulations are conducted to support our theoretical results. A brief conclusion is given in the last section.

\section{Existence of Unique Positive Global Solution}

In this section, by structuring Lyapunov function, we prove the existence of a unique global positive solution for the stochastic system (2).

Theorem 1. For any initial value $(S(0), I(0), R(0)) \in \mathbb{R}_{+}^{3}$, there exists a unique positive solution $(S(t), I(t), R(t))$ of system (2) on $t \geq 0$ and the solution will remain in $\mathbb{R}_{+}^{3}$ with probability one, i.e. $(S(t), I(t), R(t)) \in \mathbb{R}_{+}^{3}$ for all $t \geq 0$ almost surely (a.s.). 
Proof. Since the coefficients of system (2) are locally Lipschitz continuous, then for any initial value $(S(0), I(0), R(0)) \in \mathbb{R}_{+}^{3}$ there is a unique local solution $(S(t), I(t), R(t))$ on $t \in\left[0, \tau_{e}\right)$, where $\tau_{e}$ is the explosion time [14]. Now, let us show that this solution is global, i.e., $\tau_{e}=\infty$ a.s.. Let $n_{0}>0$ be sufficiently large such that $S(0), I(0)$ and $R(0)$ lie within the interval $\left[\frac{1}{n_{0}}, n_{0}\right]$. For each integer $n \geq n_{0}$, define the stopping-times

$$
\tau_{n}=\inf \left\{t \in\left[0, \tau_{e}\right): \min \{S(t), I(t), R(t)\} \leq \frac{1}{n} \text { or } \max \{S(t), I(t), R(t)\} \geq n\right\} .
$$

Set $\inf \emptyset=\infty$ ( $\emptyset$ represents the empty set $)$, and $\tau_{n}$ is increasing as $n \rightarrow \infty$. Let $\tau_{\infty}=\lim _{n \rightarrow \infty} \tau_{n}$, then $\tau_{\infty} \leq \tau_{e}$ a.s.. The next task is to show $\tau_{\infty}=\infty$ a.s.. If this statement is violated, then there exist a constant $T>0$ and $\epsilon \in(0,1)$ such that $\mathbb{P}\left\{\tau_{\infty} \leq T\right\}>\epsilon$. As a consequence, there exists an integer $n_{1} \geq n_{0}$ such that

$$
\mathbb{P}\left\{\tau_{n} \leq T\right\} \geq \epsilon \text { for all } n \geq n_{1} .
$$

Denote $N(t)=S(t)+I(t)+R(t)$, then $d N(t)=(b-d N(t)) d t$. Thus $\lim _{t \rightarrow \infty} N(t) \leq N_{0}$ a.s. for all $t \in\left[0, \tau_{n}\right]$, where $N_{0}=\max \left\{N(0), \frac{b}{d}\right\}$.

Define a $C^{2}$-function $V: \mathbb{R}_{+}^{3} \rightarrow \mathbb{R}_{+}$by

$$
V(t)=V(S, I, R)=S-1-\ln S+I-1-\ln I+R-1-\ln R .
$$

Since $u-1-\ln u \geq 0$ for any $u>0$, it is clear that $V(t)$ is nonnegative.

Let $n \geq n_{0}$ and $T>0$ be arbitrary. Applying Itô's formula to $V(t)$, we obtain that

$$
d V(S, I, R)=\mathcal{L} V d t-\left(1-\frac{1}{S}\right) \frac{\sigma S I^{2}}{1+k I+\alpha I^{2}} d B(t)+\left(1-\frac{1}{I}\right) \frac{\sigma S I^{2}}{1+k I+\alpha I^{2}} \mathrm{~d} B(t),
$$

where

$$
\begin{aligned}
\mathcal{L} V & =\left(1-\frac{1}{S}\right)\left(b-d S-\frac{\beta S I^{2}}{1+k I+\alpha I^{2}}+\delta R\right)+\left(1-\frac{1}{I}\right)\left(\frac{\beta S I^{2}}{1+k I+\alpha I^{2}}-(d+\mu) I\right) \\
& +\left(1-\frac{1}{R}\right)(\mu I-(d+\delta) R)+\frac{\sigma^{2} I^{4}}{2\left(1+k I+\alpha I^{2}\right)^{2}}+\frac{\sigma^{2} S^{2} I^{2}}{2\left(1+k I+\alpha I^{2}\right)^{2}} \\
& =b+3 d+\mu+\delta+\frac{\beta I^{2}}{1+k I+\alpha I^{2}}+\frac{\sigma^{2} I^{4}}{2\left(1+k I+\alpha I^{2}\right)^{2}}+\frac{\sigma^{2} S^{2} I^{2}}{2\left(1+k I+\alpha I^{2}\right)^{2}} \\
& -d S-d I-d R-\frac{b}{S}-\frac{\delta R}{S}-\frac{\mu I}{R}-\frac{\beta S I}{1+k I+\alpha I^{2}} \\
& \leq b+3 d+\mu+\delta+\frac{\beta}{\alpha}+\frac{\sigma^{2}}{2 \alpha^{2}}+\frac{\sigma^{2} N_{0}^{2}}{2 k^{2}}:=K .
\end{aligned}
$$

Substitute this inequality into (5), and we have

$$
d V(S, I, R) \leq K d t-(S-1) \frac{\sigma I^{2}}{1+k I+\alpha I^{2}} d B(t)+(I-1) \frac{\sigma S I}{1+k I+\alpha I^{2}} \mathrm{~d} B(t) .
$$

Integrating both sides of the above inequality from 0 to $\tau_{n} \bigwedge T$ and taking the expectation yield

$$
E V\left(S\left(\tau_{n} \wedge T\right), I\left(\tau_{n} \wedge T\right), R\left(\tau_{n} \wedge T\right)\right) \leq V(S(0), I(0), R(0))+K T .
$$

Set $\Omega_{n}=\left\{\tau_{n} \leq T\right\}$ for $n \geq n_{1}$, and we have $\mathbb{P}\left(\Omega_{k}\right) \geq \epsilon$ according to (4). Note that for every $\omega \in \Omega_{n}$, there is at least one of $S\left(\tau_{n}, \omega\right), I\left(\tau_{n}, \omega\right), R\left(\tau_{n}, \omega\right)$ that equals to either $n$ or $\frac{1}{n}$, then $V\left(S\left(\tau_{n}\right), I\left(\tau_{n}\right), R\left(\tau_{n}\right)\right)$ equals to either $n$ or $\frac{1}{n}$. Hence

$$
V\left(S\left(\tau_{n} \wedge T\right), I\left(\tau_{n} \wedge T\right), R\left(\tau_{n} \wedge T\right)\right) \geq(n-1-\ln n) \wedge\left(\frac{1}{n}-1-\ln \frac{1}{n}\right)
$$


Following from (6), we have

$$
\begin{aligned}
V(S(0), I(0), R(0))+K T & \geq E\left[I_{\Omega_{n}(\omega)} V\left(S\left(\tau_{n}\right), I\left(\tau_{n}\right), R\left(\tau_{n}\right)\right)\right] \\
& \geq \varepsilon\left((n-1-\ln n) \wedge\left(\frac{1}{n}-1-\ln \frac{1}{n}\right)\right),
\end{aligned}
$$

where $I_{\Omega_{n}(\omega)}$ is the indicator function of $\Omega_{n}$. Taking $n \rightarrow \infty$ yields that $\infty>V(S(0), I(0), R(0))+K T>$ $+\infty$, which is a contradiction. The conclusion is confirmed.

Based on the above theorem, we present the following two lemmas for later use.

Lemma 2. ([13]) The plane $S+I+R=\frac{b}{d}$ is an invariant manifold of system (1), which is attracting in the first octant.

Lemma 3. For any positive solution $(S(t), I(t), R(t))$ of system (2) with initial value $(S(0), I(0), R(0)) \in$ $\mathbb{R}_{+}^{3}$, we have

$$
\max \left\{\limsup _{t \rightarrow \infty} S(t), \limsup _{t \rightarrow \infty} I(t), \limsup _{t \rightarrow \infty} R(t)\right\} \leq \frac{b}{d} \quad \text { a.s. }
$$

Proof. Sum up the three equations in (2) and denote $N(t)=S(t)+I(t)+R(t)$, then we have

$$
\frac{d N}{d t}=b-d N
$$

Combining with Lemma 2 implies that

$$
\lim _{t \rightarrow \infty}(S(t)+I(t)+R(t))=\frac{b}{d},
$$

then obviously we have

$$
\limsup _{t \rightarrow \infty} S(t) \leq \frac{b}{d}, \quad \limsup _{t \rightarrow \infty} I(t) \leq \frac{b}{d}, \quad \limsup _{t \rightarrow \infty} R(t) \leq \frac{b}{d} \text { a.s.. }
$$

since $S(t) \geq 0, I(t) \geq 0, R(t) \geq 0$ a.s.. This completes the proof.

\section{Disease Extinction for System (2)}

In this section, we will study the disease-free dynamics for the stochastic model (2). Denote

$$
R_{0}^{s}=\frac{\beta b}{k d(d+\mu)}-\frac{\sigma^{2} b^{2}}{2 k^{2} d^{2}(d+\mu)},
$$

which can be seen as a threshold of the extinction (i.e., disease-free) or persistence (i.e., endemic) of disease for the stochastic model. For the sake of simplicity, we denote the following two conditions:

$$
(C 1) R_{0}^{s}<1 \text { and } \sigma^{2} \leq \frac{\beta k d}{b}, \quad(C 2) \sigma^{2} \geq \max \left\{\frac{\beta k d}{b}, \frac{\beta^{2}}{2(d+\mu)}\right\} .
$$

Theorem 4. Let $(S(t), I(t), R(t))$ be the solution of system (2) with any initial value $(S(0), I(0), R(0)) \in$ $\mathbb{R}_{+}^{3}$. If condition $(C 1)$ or $(C 2)$ holds, then the disease dies out with probability one. And the solution $(S(t), I(t), R(t))$ of model (2) has the following property:

$$
\begin{aligned}
& \lim _{t \rightarrow \infty} S(t)=\frac{b}{d} \quad \text { a.s. } \\
& \lim _{t \rightarrow \infty} I(t)=0 \quad \text { a.s. } \\
& \lim _{t \rightarrow \infty} R(t)=0 \quad \text { a.s. }
\end{aligned}
$$


Proof. Applying the Itô's formula to $\ln I(t)$ yields

$$
\begin{aligned}
d \ln I(t) & =\left(\frac{\beta S I}{1+k I+\alpha I^{2}}-(d+\mu)-\frac{\sigma^{2} S^{2} I^{2}}{2\left(1+k I+\alpha I^{2}\right)^{2}}\right) d t+\frac{\sigma S I}{1+k I+\alpha I^{2}} d B(t) \\
& =\left[-\frac{\sigma^{2}}{2}\left(\frac{S I}{1+k I+\alpha I^{2}}-\frac{\beta}{\sigma^{2}}\right)^{2}+\frac{\beta^{2}}{2 \sigma^{2}}-(d+\mu)\right] d t+\frac{\sigma S I}{1+k I+\alpha I^{2}} d B(t) \\
& :=\phi\left(\frac{S I}{1+k I+\alpha I^{2}}\right) d t+\frac{\sigma S I}{1+k I+\alpha I^{2}} d B(t),
\end{aligned}
$$

where $\phi: \mathbb{R}_{+}^{2} \rightarrow \mathbb{R}$ is defined by $\phi(x)=-\frac{\sigma^{2} x^{2}}{2}+\beta x-(d+\mu)$.

Integrate both sides of the above equation from 0 to $t$, and we get

$$
\ln I(t)=\ln I(0)+\int_{0}^{t} \phi\left(\frac{S(r) I(r)}{1+k I(r)+\alpha I^{2}(r)}\right) d r+\int_{0}^{t} \frac{\sigma S(r) I(r)}{1+k I(r)+\alpha I^{2}(r)} d B(r) .
$$

Set $G(t)=\int_{0}^{t} \frac{\sigma S(r) I(r)}{1+k I(r)+\alpha I^{2}(r)} d B(r)$, then by the strong law of large numbers for martingales [14], we have

$$
\lim _{t \rightarrow \infty} \frac{G(t)}{t}=0 \text { a.s.. }
$$

If the condition $(C 2)$ holds, then we get

$$
\phi\left(\frac{S I}{1+k I+\alpha I^{2}}\right)=-\frac{\sigma^{2}}{2}\left(\frac{S I}{1+k I+\alpha I^{2}}-\frac{\beta}{\sigma^{2}}\right)^{2}+\frac{\beta^{2}}{2 \sigma^{2}}-(d+\mu) \leq \frac{\beta^{2}}{2 \sigma^{2}}-(d+\mu) .
$$

According to (7), we have

$$
\frac{\ln I(t)}{t} \leq \frac{\ln I(0)}{t}+\frac{1}{t} \int_{0}^{t}\left(\frac{\beta^{2}}{2 \sigma^{2}}-(d+\mu)\right) d r+\frac{G(t)}{t}
$$

Taking the limit superior of both sides of $(7)$, we can obtain

$$
\limsup _{t \rightarrow \infty} \frac{\ln I(t)}{t} \leq \frac{\beta^{2}}{2 \sigma^{2}}-(d+\mu)<0 \quad \text { a.s.. }
$$

which implies $\lim _{t \rightarrow \infty} I(t)=0$ a.s..

Now we consider the condition $(C 1)$. In this case,

$$
\begin{aligned}
\phi\left(\frac{S I}{1+k I+\alpha I^{2}}\right) & =-\frac{\sigma^{2}}{2}\left(\frac{S I}{1+k I+\alpha I^{2}}-\frac{\beta}{\sigma^{2}}\right)^{2}+\frac{\beta^{2}}{2 \sigma^{2}}-(d+\mu) \\
& \leq-\frac{\sigma^{2}}{2}\left(\frac{b}{k d}-\frac{\beta}{\sigma^{2}}\right)^{2}+\frac{\beta^{2}}{2 \sigma^{2}}-(d+\mu) \\
& =\frac{\beta b}{k d}-\frac{\sigma^{2} b^{2}}{2 k^{2} d^{2}}-(d+\mu) \\
& =(d+\mu)\left(\frac{\beta b}{k d(d+\mu)}-\frac{\sigma^{2} b^{2}}{2 k^{2} d^{2}(d+\mu)}-1\right) \\
& =(d+\mu)\left(R_{0}^{s}-1\right) .
\end{aligned}
$$

From (7), we have

$$
\frac{\ln I(t)}{t} \leq \frac{\ln I(0)}{t}+(d+\mu)\left(R_{0}^{s}-1\right)+\frac{1}{t} G(t)
$$


According to condition $(C 1)$, one can get

$$
\limsup _{t \rightarrow \infty} \frac{\ln I(t)}{t} \leq(d+\mu)\left(R_{0}^{s}-1\right)<0 \quad \text { a.s.. }
$$

which implies $\lim _{t \rightarrow \infty} I(t)=0$ a.s..

Without loss of generality, let $\bar{\Omega}=\left\{\omega \in \Omega: \lim _{t \rightarrow \infty} I(t)=0\right\}$, then we have $\mathbb{P}(\bar{\Omega})=1$. Hence, for any $\omega \in \bar{\Omega}$ and any sufficiently small constant $\varepsilon_{1}>0$, there exists $T\left(\omega, \varepsilon_{1}\right)>0$ such that $I(\omega, t) \leq \varepsilon_{1}$ for all $t \geq T$.

Then from the third equation of model (2), we have

$$
d R(\omega, t) \leq\left[\mu \varepsilon_{1}-(d+\delta) R(\omega, t)\right] d t, \quad \text { for all } \omega \in \bar{\Omega}, t \geq T .
$$

By the comparison theorem, it follows that

$$
\limsup _{t \rightarrow \infty} R(\omega, t) \leq \frac{\mu \varepsilon_{1}}{d+\delta}, \quad \text { for all } \omega \in \bar{\Omega} .
$$

Note that $R(\omega, t)>0$ for almost all $\omega \in \Omega$ and $t>0$, and let $\varepsilon_{1} \rightarrow 0$, then we have

$$
\lim _{t \rightarrow \infty} R(\omega, t)=0, \quad \omega \in \bar{\Omega} .
$$

Recall that $\mathbb{P}(\bar{\Omega})=1$. Therefore, $\lim _{t \rightarrow \infty} R(t)=0$ a.s..

Since $I(\omega, t) \leq \varepsilon_{1}$ for all $t \geq T$ and $\omega \in \bar{\Omega}$, then by the first equation of system (2), we have

$$
\begin{aligned}
\frac{d S(t)}{d t} & \geq b-\left(d+\frac{\beta I^{2}}{1+k I+\alpha I^{2}}+\frac{\sigma I^{2}}{1+k I+\alpha I^{2}}|\dot{B}(t)|\right) S \\
& \geq b-\left(d+\frac{\beta \varepsilon_{1}}{k}+\frac{\sigma \varepsilon_{1}}{k}|\dot{B}(t)|\right) S .
\end{aligned}
$$

As $\varepsilon_{1} \rightarrow 0$, taking the inferior limit of both sides of (8) yields

$$
\liminf _{t \rightarrow \infty} S(t) \geq \frac{b}{d} \quad \text { a.s.. }
$$

By the proof of Lemma 3, we have

$$
\limsup _{t \rightarrow \infty} S(t) \leq \frac{b}{d} \quad \text { a.s.. }
$$

From (9) and (10), we have

$$
\lim _{t \rightarrow \infty} S(t)=\frac{b}{d} \quad \text { a.s.. }
$$

The proof is finished.

\section{Asymptotic Behavior around the Disease-Free Equilibrium}

In this section, we study the asymptotic behavior of the stochastic system (2) near the disease-free equilibrium $E_{0}$ of deterministic system (1), by estimating the distance between the stochastic solution and the disease-free equilibrium in the sense of time mean.

Theorem 5. Let $(S(t), I(t), R(t))$ be the solution of system (2) with any initial value $(S(0), I(0), R(0)) \in$ $\mathbb{R}_{+}^{3}$. If $\frac{\beta b}{k d(d+\mu)} \leq 1, d \alpha^{2}>2 \sigma^{2}$, and $M_{1}>0$, then

$$
\limsup _{t \rightarrow \infty} \frac{1}{t} E \int_{0}^{t}\left[\frac{3 d}{4}\left(S(r)-\frac{b}{d}\right)^{2}+\frac{d+\mu}{8} I^{2}(r)+M_{1} R^{2}(r)\right] d t \leq M
$$


where

$$
M=\frac{\sigma^{2}(2 d+\mu)^{2} b^{2}}{d^{2}(d+\mu)\left(d \alpha^{2}-2 \sigma^{2}\right)}, M_{1}=\frac{d^{2}(d+\mu)}{8 \mu}-\frac{(2 d+\mu) \delta^{2}}{d(d+\mu)}-\frac{\alpha^{2} \delta^{2}(2 d+\mu)^{2}}{2 d(d+\mu)\left(d \alpha^{2}-2 \sigma^{2}\right)}
$$

Proof. Define the following $C^{2}$ functions

$$
V_{1}(S)=\frac{1}{2}\left(S-\frac{b}{d}\right)^{2}, \quad V_{2}(I)=\frac{b}{d} I, \quad V_{3}(S, I)=\frac{1}{2}\left(S-\frac{b}{d}+I\right)^{2}, \quad V_{4}(R)=\frac{R^{2}}{2} .
$$

Applying Itô's formula to $V_{1}(S)$, we have

$$
\begin{aligned}
d V_{1} & =\left[\left(S-\frac{b}{d}\right)\left(b-d S-\frac{\beta S I^{2}}{1+k I+\alpha I^{2}}+\delta R\right)+\frac{\sigma^{2} S^{2} I^{4}}{2\left(1+k I+\alpha I^{2}\right)^{2}}\right] d t \\
& -\left(S-\frac{b}{d}\right) \frac{\sigma S I^{2}}{1+k I+\alpha I^{2}} d B(t),
\end{aligned}
$$

where

$$
\mathcal{L} V_{1}=\left(S-\frac{b}{d}\right)\left(b-d S-\frac{\beta S I^{2}}{1+k I+\alpha I^{2}}+\delta R\right)+\frac{\sigma^{2} S^{2} I^{4}}{2\left(1+k I+\alpha I^{2}\right)^{2}}
$$

By computation,

$$
\begin{aligned}
\mathcal{L} V_{1} & =-d\left(S-\frac{b}{d}\right)^{2}-\left(S-\frac{b}{d}\right) \frac{\beta S I^{2}}{1+k I+\alpha I^{2}}+\delta\left(S-\frac{b}{d}\right) R+\frac{\sigma^{2} S^{2} I^{4}}{2\left(1+k I+\alpha I^{2}\right)^{2}} \\
& =-d\left(S-\frac{b}{d}\right)^{2}-\frac{\beta I^{2}}{1+k I+\alpha I^{2}}\left(S-\frac{b}{d}\right)^{2}-\frac{\beta b I^{2}}{d\left(1+k I+\alpha I^{2}\right)}\left(S-\frac{b}{d}\right) \\
& +\delta\left(S-\frac{b}{d}\right) R+\frac{\sigma^{2} S^{2} I^{4}}{2\left(1+k I+\alpha I^{2}\right)^{2}} \\
& \leq-d\left(S-\frac{b}{d}\right)^{2}-\frac{\beta b I^{2}}{d\left(1+k I+\alpha I^{2}\right)}\left(S-\frac{b}{d}\right)+\frac{\delta^{2}}{2 d} R^{2}+\frac{d}{2}\left(S-\frac{b}{d}\right)^{2}+\frac{\sigma^{2} S^{2}}{2 \alpha^{2}} \\
& \leq-\left(\frac{d}{2}-\frac{\sigma^{2}}{\alpha^{2}}\right)\left(S-\frac{b}{d}\right)^{2}-\frac{\beta b I^{2}}{d\left(1+k I+\alpha I^{2}\right)}\left(S-\frac{b}{d}\right)+\frac{\delta^{2}}{2 d} R^{2}+\frac{\sigma^{2} b^{2}}{d^{2} \alpha^{2}},
\end{aligned}
$$

where in the above inequality, we have used the inequality $2 a b \leq a^{2}+b^{2},(a+b)^{2} \leq 2 a^{2}+2 b^{2}$ for any $a, b \in \mathbb{R}$. Similarly, we have

$$
\begin{aligned}
\mathcal{L} V_{2} & =\frac{b}{d}\left(\frac{\beta S I^{2}}{1+k I+\alpha I^{2}}-(d+\mu) I\right) \\
& =\frac{\beta b I^{2}}{d\left(1+k I+\alpha I^{2}\right)}\left(S-\frac{b}{d}\right)+\frac{\beta b^{2} I^{2}}{d^{2}\left(1+k I+\alpha I^{2}\right)}-\frac{b(d+\mu) I}{d} \\
& \leq \frac{\beta b I^{2}}{d\left(1+k I+\alpha I^{2}\right)}\left(S-\frac{b}{d}\right)+\frac{\beta b^{2} I}{d^{2} k}-\frac{b(d+\mu) I}{d} \\
& =\frac{\beta b I^{2}}{d\left(1+k I+\alpha I^{2}\right)}\left(S-\frac{b}{d}\right)+\frac{b(d+\mu)}{d}\left(\frac{\beta b}{d k(d+\mu)}-1\right) I \\
& \leq \frac{\beta b I^{2}}{d\left(1+k I+\alpha I^{2}\right)}\left(S-\frac{b}{d}\right),
\end{aligned}
$$


and

$$
\begin{aligned}
\mathcal{L} V_{3} & =\left(S-\frac{b}{d}+I\right)(b-d S+\delta R-(d+\mu) I) \\
& =-d\left(S-\frac{b}{d}\right)^{2}+\delta\left(S-\frac{b}{d}\right) R-(2 d+\mu)\left(S-\frac{b}{d}\right) I+\delta R I-(d+\mu) I^{2} \\
& \leq\left[-\frac{3 d}{4}+\frac{(2 d+\mu)^{2}}{2(d+\mu)}\right]\left(S-\frac{b}{d}\right)^{2}-\frac{d+\mu}{4} I^{2}+\left(\frac{\delta^{2}}{d}+\frac{\delta^{2}}{d+\mu}\right) R^{2},
\end{aligned}
$$

and here in the above inequality, we have used the inequality $2 a b \leq a^{2}+b^{2}$ for any $a, b \in \mathbb{R}$, and the Young inequality $-(2 d+\mu)\left(S-\frac{b}{d}\right) I \leq \frac{d+\mu}{2} I^{2}+\frac{(2 d+\mu)^{2}}{2(d+\mu)}\left(S-\frac{b}{d}\right)^{2}$.

Since $\mathcal{L} V_{4}=\mu I R-(d+\delta) R^{2}$, then we have

$$
\begin{aligned}
\frac{d(d+\mu)}{4 \mu} \mathcal{L} V_{4} & =\frac{d(d+\mu)}{4} I R-\frac{d(d+\mu)(d+\delta)}{4 \mu} R^{2} \\
& \leq \frac{d+\mu}{8} I^{2}+\frac{d^{2}(d+\mu)}{8} R^{2}-\frac{d(d+\mu)(d+\delta)}{4 \mu} R^{2} \\
& \leq \frac{d+\mu}{8} I^{2}-\frac{d^{2}(d+\mu)}{8 \mu} R^{2} .
\end{aligned}
$$

By (11) and (12), we have

$$
\mathcal{L} V_{1}+\mathcal{L} V_{2} \leq-\left(\frac{d}{2}-\frac{\sigma^{2}}{\alpha^{2}}\right)\left(S-\frac{b}{d}\right)^{2}+\frac{\delta^{2}}{2 d} R^{2}+\frac{\sigma^{2} b^{2}}{d^{2} \alpha^{2}}
$$

Consider a positive definite $C^{2}$ function $V: \mathbb{R}_{+}^{3} \rightarrow \mathbb{R}_{+}$as follows

$$
V=V_{3}+\frac{(2 d+\mu)^{2}}{2(\mu+d)\left(\frac{d}{2}-\frac{\sigma^{2}}{\alpha^{2}}\right)}\left(V_{1}+V_{2}\right)+\frac{d(\mu+d)}{4 \mu} V_{4},
$$

then by (13)-(15), we can compute

$$
\begin{aligned}
\mathcal{L} V & =\mathcal{L} V_{3}+\frac{(2 d+\mu)^{2}}{2(\mu+d)\left(\frac{d}{2}-\frac{\sigma^{2}}{\alpha^{2}}\right)}\left(\mathcal{L} V_{1}+\mathcal{L} V_{2}\right)+\frac{d(\mu+d)}{4 \mu} \mathcal{L} V_{4} \\
& \leq-\frac{3 d}{4}\left(S-\frac{b}{d}\right)^{2}-\left(\frac{d^{2}(d+\mu)}{8 \mu}-\frac{\delta^{2}(2 d+\mu)}{d(\mu+d)}-\frac{\alpha^{2} \delta^{2}(2 d+\mu)^{2}}{2 d(\mu+d)\left(d \alpha^{2}-2 \sigma^{2}\right)}\right) R^{2} \\
& -\frac{d+\mu}{8} I^{2}+\frac{\sigma^{2} b^{2}(2 d+\mu)^{2}}{d^{2}(\mu+d)\left(d \alpha^{2}-2 \sigma^{2}\right)} \\
& =-\frac{3 d}{4}\left(S-\frac{b}{d}\right)^{2}-\frac{d+\mu}{8} I^{2}-M_{1} R^{2}+M .
\end{aligned}
$$

Integrating the above inequality and taking expectation yield

$$
E V(t)-V(0) \leq-\frac{3 d}{4} E \int_{0}^{t}\left(S(r)-\frac{b}{d}\right)^{2} d r-\frac{d+\mu}{8} E \int_{0}^{t} I^{2}(r) d r-M_{1} E \int_{0}^{t} R^{2}(r) d r+M t .
$$

Hence,

$$
\limsup _{t \rightarrow \infty} \frac{1}{t} E \int_{0}^{t}\left\{\frac{3 d}{4}\left(S(r)-\frac{\mu}{\mu_{1}}\right)^{2}+\frac{d+\mu}{8} I^{2}(r)+M_{1} R^{2}(r)\right\} d r \leq M .
$$

Theorem 5 shows that the solution of system (2) oscillates around the disease-free equilibrium of system (1), and the magnitude of the oscillation is proportional to the intensity of white noise. From the perspective of biology, if the intensity of random disturbance is small enough, the disease will be controlled in a small range under certain conditions. 


\section{Stationary Distribution}

In this section, we will try to establish sufficient condition for the existence of stationary distribution for system (2). We first present the following lemma.

Lemma 6. ([4]) The Markov process X(t), the solution of system (3), has a unique ergodic stationary distribution $\pi(\cdot)$, if there exists a bounded domain $D \subset R^{l}$ with regular boundary $\Gamma$ and

(i) there is a positive number $M$ such that $\sum_{i, j=1}^{l} a_{i j}(x) \xi_{i} \xi_{j} \geq M|\xi|^{2}, x \in D, \xi \in R^{l}$,

(ii) there exists a nonnegative $C^{2}$-function $V$ such that $L V$ is negative for any $R^{l} \backslash D$. Then

$$
\mathbb{P}_{x}\left\{\lim _{T \rightarrow \infty} \frac{1}{T} \int_{0}^{T} f(X(t)) d t=\int_{\mathbb{R}^{n}} f(x) \mu(d x)\right\}=1,
$$

for all $x \in R^{l}$, where $f(\cdot)$ is a function integrable with respect to the measure $\pi$.

Theorem 7. If $R_{0}^{s}>1$, then for any positive initial value $(S(0), I(0), R(0)) \in \Gamma$, system (2) admits a unique stationary distribution $\Pi$ and the ergodicity holds, where $\Gamma=\left\{x \in(0, b / d)^{3} \mid 0<x_{1}+x_{2}+x_{3}\right.$ $\leq b / d\} \subset \mathbb{R}_{+}^{3}$.

Proof. We only need to validate the conditions $(i)$ and $(i i)$ in Lemma 6 . Now we shall prove that the conditions $(i)$ holds. Let $D=\left\{x \in \Gamma \mid \frac{1}{\alpha_{1}}<x_{1}, x_{2}<\frac{b}{d}-\frac{1}{\alpha_{1}}, \frac{1}{\alpha_{2}}<x_{3}<\frac{b}{d}-\frac{1}{\alpha_{2}}\right.$ and $\left.\alpha_{1}<\alpha_{2}\right\}$, where $\alpha_{1}, \alpha_{2}$ are sufficiently large positive constants to be chosen in the following. The diffusion matrix of system (2) is given by

$$
A=\left(\frac{\sigma S I^{2}}{1+k I+\alpha I^{2}}\right)^{2}\left[\begin{array}{ccc}
1 & -1 & 0 \\
-1 & 1 & 0 \\
0 & 0 & 0
\end{array}\right]
$$

We have

$$
a_{11}(S, I, R)=\left(\frac{\sigma S I^{2}}{1+k I+\alpha I^{2}}\right)^{2} \geq \min _{(S, I, R) \in \bar{D} \subset \mathbb{R}_{+}^{3}}\left\{\left(\frac{\sigma S I^{2}}{1+k I+\alpha I^{2}}\right)^{2}\right\} \geq \lambda
$$

where $\lambda$ is a positive constant. Then the conditions $(i)$ in Lemma 6 is satisfied.

Secondly, we verify the conditions $(i i)$. Define the a $C^{2}$ function as follows

$$
V(S, I, R)=V_{1}(S, I)+V_{2}(I)+V_{3}(R),
$$

where

$$
V_{1}(S, I)=\frac{1}{S}+\frac{1}{\theta} I^{-\theta}\left(\frac{b}{d}-S\right), V_{2}(I)=\frac{1}{\theta} I^{-\theta}, V_{3}(R)=R-\ln R+\rho,
$$

and $\theta$ is a positive constant to be chosen later and $\rho>0$ is so large that $V_{3}(R) \geq 0$ for all $R>0$. By the Itô's formula, we obtain

$$
\begin{aligned}
\mathcal{L} V_{1} & =\left(-\frac{1}{S^{2}}-\frac{1}{\theta} I^{-\theta}\right)\left(b-d S-\frac{\beta S I^{2}}{1+k I+\alpha I^{2}}+\delta R\right)+\frac{\sigma^{2} I^{4}}{\left(1+k I+\alpha I^{2}\right)^{2} S} \\
& -I^{-\theta-1}\left(\frac{b}{d}-S\right)\left(\frac{\beta S I^{2}}{1+k I+\alpha I^{2}}-(d+\mu) I\right)+\frac{(\theta+1) \sigma^{2} S^{2} I^{4} I^{-\theta-2}}{2\left(1+k I+\alpha I^{2}\right)^{2}}\left(\frac{b}{d}-S\right) \\
& -\frac{\sigma^{2} S^{2} I^{4}}{\left(1+k I+\alpha I^{2}\right)^{2}} I^{-\theta-1} \\
& \leq\left[-\frac{d}{\theta}+d+\mu+\frac{(\theta+1) \sigma^{2} b^{2}}{2 k^{2} d^{2}}\right]\left(\frac{b}{d}-S\right) I^{-\theta}-\frac{b}{S^{2}}+\frac{1}{S}\left(d+\frac{\beta b}{k d}+\frac{\sigma^{2} b^{2}}{k^{2} d^{2}}\right)+\frac{\beta b}{k d \theta} I^{1-\theta} \\
& \leq\left[-\frac{d}{\theta}+d+\mu+\frac{(\theta+1) \sigma^{2} b^{2}}{2 k^{2} d^{2}}\right]\left(\frac{b}{d}-S\right) I^{-\theta}-\frac{b}{2 S^{2}}+\frac{1}{2 b}\left(d+\frac{\beta b}{k d}+\frac{\sigma^{2} b^{2}}{k^{2} d^{2}}\right)^{2}+\frac{\beta b I^{1-\theta}}{k d \theta} .
\end{aligned}
$$


Similarly, we can compute that

$$
\begin{aligned}
\mathcal{L} V_{2} & =-I^{-\theta-1}\left(\frac{\beta S I^{2}}{1+k I+\alpha I^{2}}-(d+\mu) I\right)+\frac{(\theta+1) \sigma^{2} S^{2} I^{4} I^{-\theta-2}}{2\left(1+k I+\alpha I^{2}\right)^{2}} \\
& =I^{-\theta}\left[d+\mu-\frac{\beta b}{k d}+\beta\left(\frac{b}{k d}-\frac{S I}{1+k I+\alpha I^{2}}\right)+\frac{(\theta+1) \sigma^{2} S^{2} I^{2}}{2\left(1+k I+\alpha I^{2}\right)^{2}}\right] \\
& \leq I^{-\theta}\left[d+\mu-\frac{\beta b}{k d}+\beta\left(\frac{b}{k d}-\frac{S I}{1+k I+\alpha I^{2}}\right)+\frac{(\theta+1) \sigma^{2} b^{2}}{2 k^{2} d^{2}}\right] \\
& \leq I^{-\theta}\left[-\phi\left(\frac{b}{k d}\right)+\frac{\theta \sigma^{2} b^{2}}{2 k^{2} d^{2}}\right]+\frac{\beta I^{-\theta}}{k}\left(\frac{b}{d}-S\right)+\frac{\beta}{k}\left(\frac{b}{d}\right)^{1-\theta},
\end{aligned}
$$

and

$$
\mathcal{L} V_{3}=\left(1-\frac{1}{R}\right)(\mu I-(d+\delta) R) \leq-\frac{\mu I}{R}+d+\delta+\mu I \leq-\frac{\mu I}{R}+d+\delta+\frac{\mu b}{d} .
$$

Here the above inequality is derived by applying the elementary inequalities $2 a b \leq a^{2}+b^{2},(a+b)^{2} \leq$ $2 a^{2}+2 b^{2}$ for any $a, b \in \mathbb{R}$. Now we choose $\theta$ sufficiently small such that

$$
-\frac{d}{\theta}+d+\mu+\frac{\beta}{k}+\frac{(\theta+1)}{2} \frac{\sigma^{2} b^{2}}{k^{2} d^{2}}<0,
$$

and

$$
-\phi\left(\frac{b}{k d}\right)+\frac{\theta \sigma^{2} b^{2}}{2 k^{2} d^{2}}<0,
$$

where the second inequality is well-defined due to the condition $R_{0}^{s}>1$. Therefore, combining (16)-(18) leads to

$$
\begin{aligned}
\mathcal{L} V & \leq\left[-\frac{d}{\theta}+d+\mu+\frac{\beta}{k}+\frac{(\theta+1)}{2} \frac{\sigma^{2} b^{2}}{k^{2} d^{2}}\right]\left(\frac{b}{d}-S\right) I^{-\theta}+I^{-\theta}\left[-\phi\left(\frac{b}{k d}\right)+\frac{\theta \sigma^{2} b^{2}}{2 k^{2} d^{2}}\right]+\frac{\beta b I^{1-\theta}}{k d \theta} \\
& -\frac{\mu I}{R}-\frac{b}{2 S^{2}}+\varepsilon,
\end{aligned}
$$

where $\varepsilon$ is some positive constant.

It is obvious to see from (19) that there exists a big enough number $\alpha_{1}>0$, such that

$$
\mathcal{L} V \leq-1, \text { for all } S \leq \frac{1}{\alpha_{1}} \text { or } I \leq \frac{1}{\alpha_{1}} .
$$

It remains to consider the case where $S>\frac{1}{\alpha_{1}}, I>\frac{1}{\alpha_{1}}$ and $R \leq \frac{1}{\alpha_{2}}$. Since $I>\frac{1}{\alpha_{1}}$, we get

$$
\mathcal{L} V \leq-\frac{\mu}{\alpha_{1} R}+\varepsilon .
$$

This shows that there exists a sufficiently large $\alpha_{2}>\alpha_{1}>0$, such that

$$
\mathcal{L} V \leq-1, \text { for all } S>\frac{1}{\alpha_{1}}, I>\frac{1}{\alpha_{1}} \text { and } R \leq \frac{1}{\alpha_{2}} .
$$

Altogether, we have shown that $\mathcal{L} V \leq-1$ for all $x \in \bar{D}$. So the condition (ii) of Lemma 6 is met. This completes the proof.

\section{Numerical Simulations}

In order to illustrate our theoretical results, we will give some examples of numerical simulation in this section. To begin with, following Milstein's higher order method in [5], we obtain the corresponding 
(a)

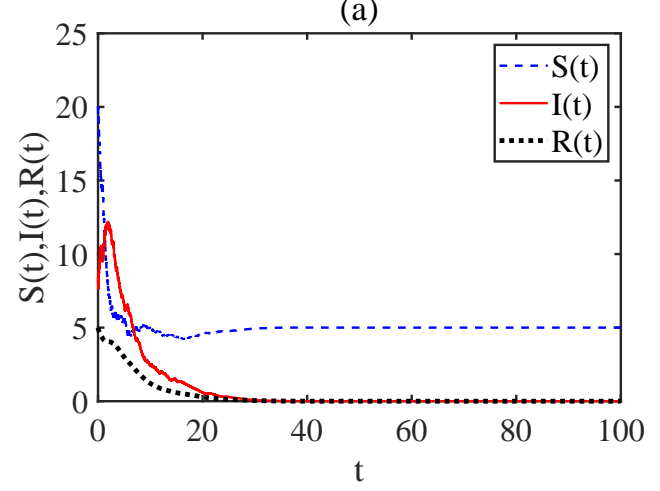

(b)

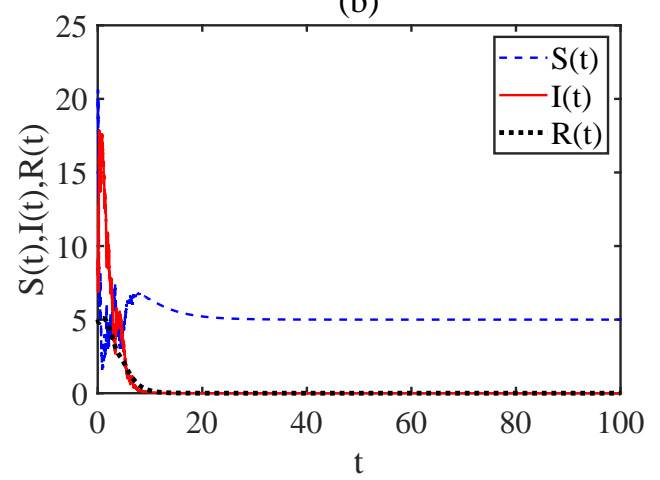

Figure 1. The solution of the stochastic system (2). (a) $\sigma=0.1$. (b) $\sigma=0.71$.

discrete equations of the stochastic system (2) as follows

$$
\left\{\begin{array}{l}
S_{i+1}=S_{i}+\left(b-d S_{i}-\frac{\beta S_{i} I_{i}^{2}}{1+k I_{i}+\alpha I_{i}^{2}}+\delta R_{i}\right) \triangle t-\frac{\sigma S_{i} I_{i}^{2} \xi_{i} \sqrt{\triangle t}}{1+k I_{i}+\alpha I_{i}^{2}}-\frac{\sigma^{2} S_{i} I_{i}^{2}\left(\xi_{i}^{2}-1\right) \Delta t}{2\left(1+k I_{i}+\alpha I_{i}^{2}\right)} \\
I_{i+1}=I_{i}+\left(\frac{\beta S_{i} I_{i}^{2}}{1+k I_{i}+\alpha I_{i}^{2}}-(d+\mu) I_{i}\right) \triangle t+\frac{\sigma S_{i} I_{i}^{2} \xi_{i} \sqrt{\triangle t}}{1+k I_{i}+\alpha I_{i}^{2}}+\frac{\sigma^{2} S_{i} I_{i}^{2}\left(\xi_{i}^{2}-1\right) \triangle t}{2\left(1+k I_{i}+\alpha I_{i}^{2}\right)} \\
R_{i+1}=R_{i}+\left(\mu I_{i}-(d+\delta) R_{i}\right) \triangle t
\end{array}\right.
$$

where the time increment $\triangle t>0$, and $\xi_{i}, i=1,2,3, \ldots, n$ are independent Gaussian random variables in $N(0,1)$. Here we choose the initial value $(S(0), I(0), R(0))=(20,8,5)$.

Example 1. Choose the value of parameters $b=1, d=0.2, \beta=0.4, k=5, \alpha=0.5, \delta=0.4, \mu=$ 0.2. To display the effect of noise intensity, we chose two different values of $\sigma$ as 0.1 (see Fig. 1 (a)) and 0.71 (see Fig. 1 (b)). In theses two cases, the condition of theorem 4 is satisfied, and the disease will die out with probability one. We can also find that when the intensity of white noise increases, the disease will disappear more rapidly.

Example 2. Choose the value of parameters $b=1, d=0.3, \beta=0.4, k=5, \alpha=0.8, \delta=0.001, \mu=$ 0.01 . The solution of deterministic system (1) tends to $E_{0}$ (see Fig. 2(a)). We chose one noise value of $\sigma$ as 0.3 (see Fig. 2(b)). According to Theorem 5, all the positive solutions of system (2) will fluctuate around the solution of system (1).

Example 3. Choose the value of parameters $b=0.9, d=0.1, \beta=0.6, k=2, \alpha=0.1, \delta=$ 0.01, $\mu=0.08$, and the noise intensity $\sigma=0.4$. Numerical simulation of the solutions is shown in Fig. 3, in which we can see that the solution of the stochastic system vibrates around the endemic equilibrium and the disease is persistent. According to Theorem 7, in this case the stochastic system (2) has a unique stationary distribution. The histograms of $S(t), I(t), R(t)$ are displayed in Fig. 4, which clearly support the result.

\section{Conclusion}

In this paper, we study a SDE version of a SIRS epidemic model with non-monotone and saturated incidence rate. Since most systems in the real world are perturbed with random factors, we introduce white noise to the transmission coefficient of the model. The existence and uniqueness of the global positive solution of the stochastic SIRS epidemic model is proved. Then the sufficient condition for the disease to disappear with probability 1 is obtained, and the dynamic behavior of the stochastic system near the disease-free equilibrium is analyzed. From the viewpoint of biology, it means that when the disturbance intensity of white noise is small enough, the disease will be controlled in a small range. Moreover, the stochastic system has a unique ergodic stationary distribution and the disease is persistent if the threshold number $R_{0}^{s}>1$. Finally, we use numerical simulations to illustrate our theoretical results. 
(a)

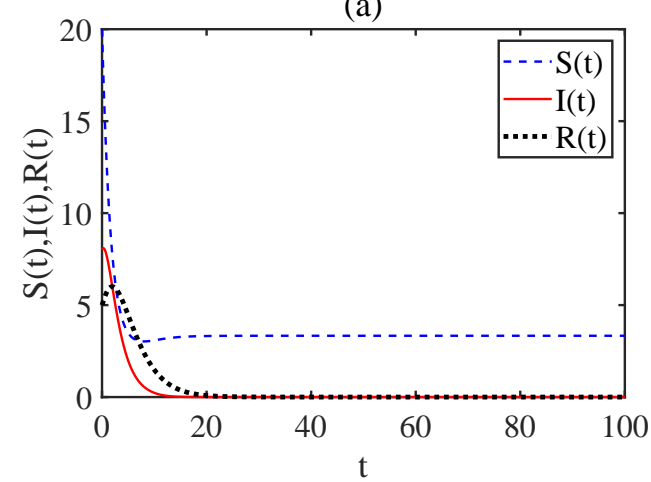

(b)

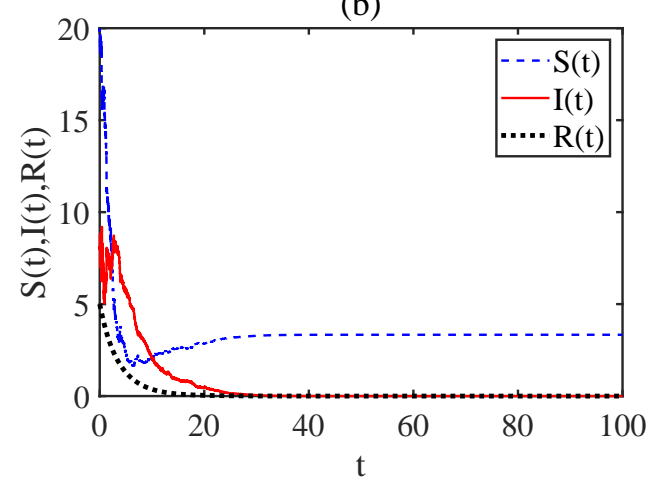

Figure 2. (a) The solution of the deterministic system (1). (b) The solutions of the stochastic system (2), where $\sigma=0.3$.

(a)

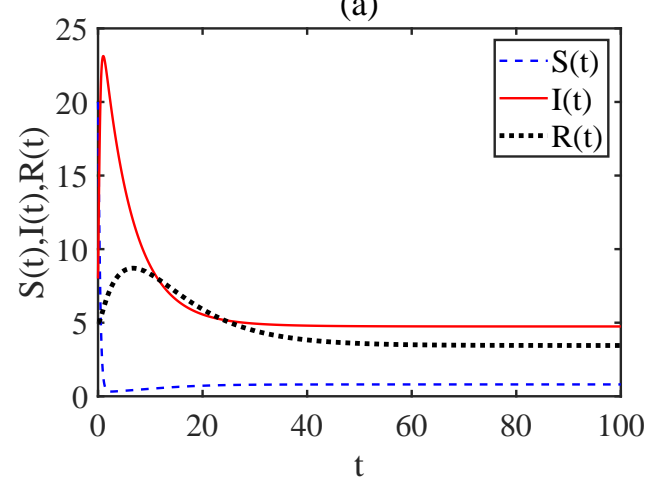

(b)

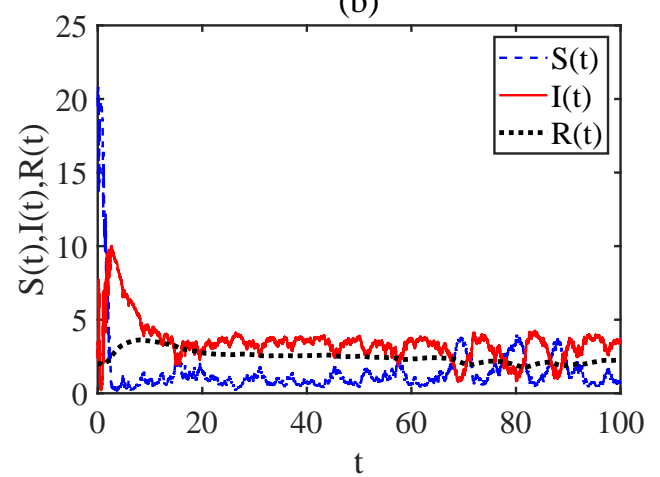

Figure 3. (a) The solution of the deterministic system (1). (b) The solution of the stochastic system (2), where $\sigma=0.4$.
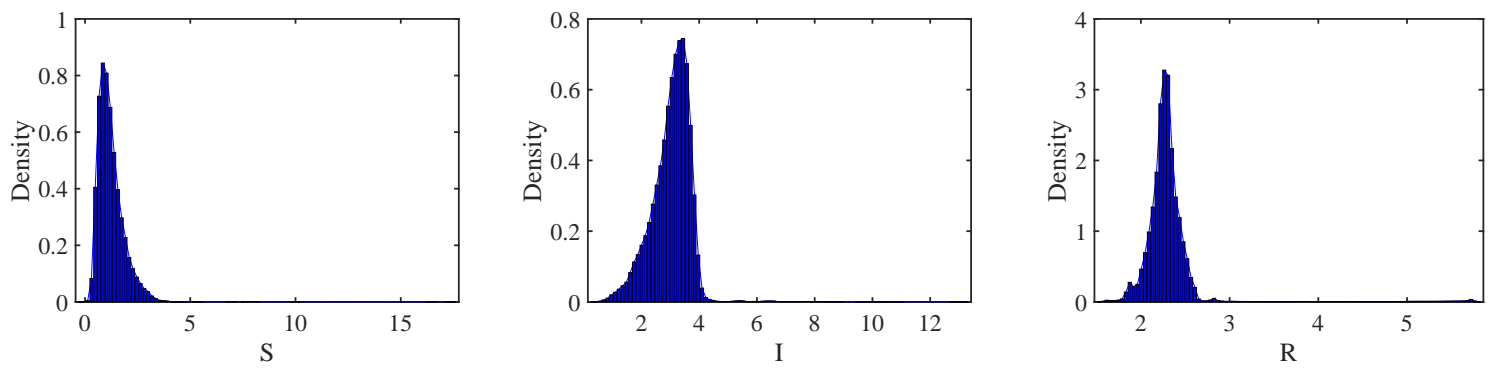

Figure 4. The histograms of the solution $(S(t), I(t), R(t))$ of the stochastic system (2) with the environmental white noise intensity $\sigma_{1}=0.4$. 
Some interesting topics deserve further consideration. Due to the square term in the saturated incidence function, the reproduction number is difficult to derive for the deterministic $S I R S$ epidemic model, and several bifurcation phenomena have been investigated in [13]. It is interesting to study the bifurcation for the stochastic model in the future research.

Acknowledgments. This work was supported by the National Natural Science Foundation of China (No. 11501364) and the Development Project of Science and Technology of University of Shanghai for Science and Technology (No. 2018KJFZ148 and No. 2019KJFZ171).

\section{References}

1. Y. Cai, Y. Kang, M. Banerjee, and W. Wang, "A stochastic SIRS epidemic model with infectious force under intervention strategies," Journal of differential equations, vol. 259, pp. 7463-7502, 2015.

2. V. Capasso and G. Serio, "A generalization of the Kermack-McKendrickdeterministic epidemic model," Mathematical Biosciences, vol. 42, pp. 43-61, 1978.

3. D. Foster and P. Young, "Stochastic evolutionary game dynamics," Handbook of Game Theory with Economic Applications, vol. 38, no. 2, pp. 219-232, 2010.

4. R. Z. Hasminskii, Stochastic Stability of Differential Equations. Sijthoff Noordhoff, 1980.

5. D. Higham, "An algorithmic introduction to numerical simulation of stochastic differential equations," SIAM Review, vol. 43, no. 3, pp. 525-546, 2001.

6. Y. Jin, W. Wang, and S. Xiao, "An sirs model with a nonlinear incidence rate," Chaos, Solitons and Fractals, vol. 34, no. 5, pp. 1482-1497, 2007.

7. W. O. Kermack and A. G. McKendrick, "Contributions to the mathematical theory of epidemics-I," Proceedings of the royal Society A, vol. 115, pp. 700-721, 1927.

8. W. O. Kermack and A. G. McKendrick, "Contributions to the mathematical theory of epidemic," Proceedings of the royal Society A, vol. 138, no. 834, pp. 55-83, 1932.

9. A. Lahrouz, H. E. Mahjour, A. Settati, and A. Bernoussi, "Dynamics and optimal control of a non-linear epidemic model with relapse and cure," Physica A: Statistical Mechanics and its Applications, vol. 496, pp. 299-317, 2018.

10. D. Li, J. Cui, M. Liu, and S. Liu, "The evolutionary dynamics of stochastic epidemic model with nonlinear incidence rate," Bulletin of Mathematical Biology, vol. 77, no. 9, pp. 1705-1743, 2015.

11. Q. Liu and Q. Chen, "Analysis of the deterministic and stochastic SIRS epidemic models with nonlinear incidence," Physica A: Statistical Mechanics and its Applications, vol. 428, pp. 140-153, 2015.

12. Q. Liu and D. Jiang, "Stationary distribution and extinction of a stochastic SIR model with nonlinear perturbation," Applied Mathematics Letters, vol. 73, pp. 8-15, 2017.

13. M. Lu, J. Huang, S. Ruan, and P. Yu, "Bifurcation analysis of an SIRS epidemic model with a generalized nonmonotone and saturated incidence rate," Journal of differential equations, vol. 267, pp. 1859-1898, 2019.

14. X. Mao, Stochastic differential equations and applications, 2nd edn. Woodhead Publishing, 2008.

15. X. Meng, S. Zhao, T. Feng, and T. Zhang, "Dynamics of a novel nonlinear stochastic SIS epidemic model with double epidemic hypothesis," Journal of Mathematical Analysis and Applications, vol. 433, no. 1, pp. 227-242, 2016.

16. R. Xu, Z. Ma, and Z. Wang, "Global stability of a delayed sirs epidemic model with saturation incidence and temporary immunity," Computers Mathematics Applications, vol. 59, no. 9, pp. 3211-3221, 2010.

17. Q. Yang, D. Jiang, N. Shi, and C. Ji, "The ergodicity and extinction of stochastically perturbed SIR and SEIR epidemic models with saturated incidence," Journal Mathmatical Analysis and Applications, vol. 388, no. 1, pp. 248-271, 2012.

18. Y. Zhao, L. Zhang and S. Yuan, "The effect of media coverage on threshold dynamics for a stochastic SIS epidemic model," Physica A: Statistical Mechanics and its Applications, vol. 512, pp. 248-260, 2018. 